\title{
LA TRANSFORMATION CARTÉSIENNE DU CONCEPT ARISTOTÉLICIEN DE SUBSTANCE
}

Pierre Aubenque

Nous savons aujourd'hui que l'ontologie, c'est-à-dire la comprèhension de l'être, qui sous-tend explicitement ou implicitement toute philosophie, a été marquée, au cours de l'histoire de la philosophie occidentale moins par des révoluitons que par une souterraine continuité. Ainsi Heidegger peut-il aflirmer que, dans la forme que lui a imprimée la scolastique, l'ontologie grecque est passée, pour l'essenticl à travers les Disputationes metaphysicae de Suarez, dans la "métaphysique" et la philosophie transcendantale des Temps modernes". I Heidegger ajoute certes que, à partir de Descartes, la question de l"ètre a cessé d"être poséc en tant que telle, mais la détermination des "domaines ontiques particuliers qui guident désormais la problématique (l'ego cogito, de Descartes, le sujet, le moi, la raison, l'esprit, la personne)" continue de s'effectuer a travers "l'arsenal de concepts de l'ontologie traditionnelle". Ces concepts se trouvent néanmoins" transpose à des régions de l'être nouvelles et subissent de ce fait des "Jormalisations" et des "restrictions". 2

Tour en reconnaissant avec Heidegger un "correspondance" entre cet élargissement et ces transformations, au prenier abord purement formelles et négatives, on peut se demander si l'éllergisement exige la transformation ou c'est contraire une tranformation d'abord spontanéc des concepts ontologiques qui ouvre de nouveaux champs du savoir. C'est cette demière hypothèse que nous voudrions essayer de confirmer sur un exemple topique: celui de la transformation du concept aristotélicien de substace presque imperceplible dans son principe, mais qui ouvre avec Descartes des horizons entièrement nouveaux.

Il y a, au premier abord, deux définitons cartésiennes de la substance. Descartes ne peut faire appel ici à une intuition, car il n'y a pas de substance que nous puissions percevoir dans l'expérience: l'expérience est la forme sous laquelle nous nous représentons les objets d'espérience. Cette forme, cette "categorie" est largement tributaire, dans son contenu, du mot que nous employons pour l'exprimer et de la métaphore que, en l'absence d'une intuition directe. il recèle inévitablement. De fait, dans la première de ces délinitions, Descartes ne fait que commenter l'étymologie du mot latin substantia. Au livre I des Principes de la Philosophie, dans le prargraphe 51 , dont le titre annonce "ce que c'est que la substance". Descartes écrit: "Lorsque nous concevons la substance, nous concevons seulement une chose qui existe en telle façon qu'elle n'a besoin que de soi-mème pour exister". C'était là, pour la tradition aristolélicienne. sinon la définition, du moins la propiété essentielle de l'ousia entendue comme liýpokémenon. La substance est ce qui subsiste sous..., et qui sert de substrat à... Le corrélat de la substance est l'attribut. Une conséquence de cette distinction, qui remonte au chapitre $\mathrm{V}$ des Catégories d'Aristote, est que la substance, expriméc par un substantif, n'a pas besoin des attributs pour être, alors que les attributs, exprimés par des adjectils, ont besoin pour exister de reposer sur $\longrightarrow$ d'être soutenus par- une substance qui en est le sujet.

Cette propiété est formulée de plusieurs façons par Aristote. Elle correspond à ce qu'il appelle la priorité "chronologique" de l'ousia (Métaph., Z, 2, 1028 a 33): aucun des autres pri-

I Sein mal Zeit, p. 22.

2 loid. 
dicats (kategoremata) n'est "sćparé" (choriston), mais le contexte montre à l'évidence que ce terme désigne ici la capacité d'être ou d'exister "sćparément" d'autre chose: dire de la substance qu'elle est "séparée", c'est dire qu'elle seule existe indípendamment des autres, alors que les autres (en fait, les attributs) ne peuvent exister indépendamment d'elle: les attributs ne sont pas sans elle (ouk aneu toutou, 1028 a29). Cette propiété est rappelée dans un texte décisit ( $Z, 3,1029$ a 27): “la séparation et la déterminité (to choriston kai to tode ii) sont ce qui appartient au plus haut point à la substance", ceci étant dit ici pour exclure la matière de la prétention à la substantialité. Enfīn, une taçon plus positive de désigner la mëme propriétí est l'expression d'origine platonicienne "par soi", qui clez Aristote a entre autres le sens suivant: "Est par soi ce dont autre close n'est pas la cause; ainsi l'homme est par soi homme" ( $\Delta .18$, 1022 a 32-35). L'homme consideré en tant qu'il est "par soi" n'a pas d'autre cause que son humanité. En ce sens, on peut dire aussi qu"il est "scipare" (kechorismenon, 1022 a 36), c"està-dire qu'il peut exister seul sans cause estéricure, qu'il est auto-suftīsant.

Ces différentes formules ont été synthétisées par la tradition aristotélicienne. La formulation nćgative de l'auto-suffisance (ne pas avoir besoin d'autre chose), celle qui sera reprise par Descartes, se rencontre dejjà chez Bočce: "Subsiste (stibsistit) ce qui n'a pas besoin soi-méme d'accident pour pouvoir être". alors que "les accidents ne sont pas de subsistances (subsistentiae non sums)". ${ }^{3}$ Thomas d'Aquin, qui suit en cela Boèce, distingue clairement entre les deux propriétés, négative et positive, de la substance. Négativement, la substance "n'a pas besoin d'un fondement extrinsèque dans lequel elle serait soutenue ou qui lui servirait de substrat (non indigat extrinseco fundamen in quo sustentetur seu substernetur)" et c'est pourquoi on dit qu' "elle subsiste par soi et n'existe pas en autre chose (subsistere per se et non in alio existere)". Positivement, la substane est le fondement des accidents, qu'elle supporte, et en ce sens on dit qu'elle leur est sous-jacente (sub-stare). ${ }^{4}$ Thomas distingue done entre subsistance (ne pas avoir besoin de fondement extrinsèque) et substantialité proprement dite au sens de substratié (être soi-même le fondement).

Aristote ne connaissait pas une telle distinction, car pour lui la substratité n'avait pas de fonction positive particulière qui ne soit déjả sous-entendue dans la propriété de subsistance. C'est seulement à partir de Boice que la substratité entendue comme fondement introduit —ou semble introduire - un rapport de causalite entre le substrat et ce qu'il "support" ou "soutitnt". Aristote parlait seulemlent d'un rapport de dépendance non réciproque: l'accident dépend de la substance; la substance ne dépend pas de l'accident. La substance n'est pas ce par quoi l'accident est; elle est seulement "ce sans quoi" l'accident noe peut être. ${ }^{5}$

Certes, le texte déjacité de Métaph., $\Delta, 18$, semble introduire la causalité dans la détinition de la substance, mais c'est de façon purement nígative: la substance, le "par soi" n'a pas besoin pour être d'une cause estrinsique; il n'y a pas quelque chose qui soit sa cause. On pourrait en conclure qu'elle est sans cause. Mais d'autre part, l'ousia est cause de l'être pour ce dont clle est la substance $(\Delta, 18,1017$ b 15). En combinant ces deux aftirmations (la substance n'a pas de cause extérieurc, la substance elle-mème est cause), on croit pouvoir généraliser en l'appliquant à la substance le principe de causalité (tout ce qui est a une cause) selon lè syllogisme implicite suivant:

3 Liber contra Euthychem el Nestorimm, in Theological Tractate's, ed. Rand et Tester, p.88, 43 et p. $90,85$.

4 In Sem., II, dist. 35, q. 2 , art. 1.

5 Mletoph., Z, I, 1028 a 29. 
La substance a une cause

La substance n'a pas de cause autre que soi.

Donc la substance a une cause qui n'est pas autre qu'elle-mëme; elle est cause de soi.

Cette interprétation avait pu ètre suggérée par la traduction latine de Guillaume de Moerbeke, qui entend ainsi le texte cité plus haut de 1022 a 22-23: "Est secundum se [ $=$ per se] cujus non est alia causa"; il aurait fallu traduire: "cujus non est alind causa" (ce dont aucune chose n'est la cause, et non: ce qui n'a pas d'autre cause".

Le pas qui fait de la substance la causa sui, étranger à Aristote, évité par Thomas d'Aquin et même encore par Suarez, esta franchi - pour ta premiëre fois, semble-t-il— par Descartes. Dans le texte des Principes, il n'utilise pas l'expresion causa sui, mais il remarque que, si la substance est définie comme il l'a fait, à savoir ce qui n'a besoin que de soi-même pour exister. ${ }^{6}$ On pourrait penser que cette conclusion paradoxale, qui sera celle de Spinozn, selon laqqueIle seul Dieu en tant que causa sui satisfait à la définition donnée de la substance, est erronée et tient à une mauvaise interprétation de la définition, qui définit al substance par la subsistance, l'indépendance à l'égard des attributs, et non par la causalité à l'égard de soi-même, l'auto-production. Descartes semble suivre un instant cette voie, puisqu'il reconnaît qu“" "il peut y avoir de l'obscurité touchant l'explication de ce mot, $n$ 'woir besoin que de soi-même", et que la définition donnée n'est pas univoque, ayant un sens diffërent pour Dieu et pour la créature. Mais, au lieu d'analyser et de résoudre l'équivocité, Descantes conserve la délinition, en faisant seulement une exception à son application universelle: quand on parle de substances créses, on veut dire qu'elles n'ont besoin pour subsister d'aucun concours à l'exception du concours ordinaire de Dieu; elles n'ont pas besoin des qualités ou des attributs, alors, que les qualités et les attributs ont besoin du concours ordinaire de Dieu, mais aussi de la substance dont ils sont prédiqués ou dans laquelle ils inhèrent.

Descartes interprète donc bien la définition traditionnelle dans le sens de l'auto-causalité. La substance n'est pas seulement per se, mais aussi a se, au sens où ab exprime en latin un rapport de production causale. La substance n'est pas seulement autosubsistante, mais elle est autoproductrice, avec cette restriction que pour les substances créés cetteautoproduction n'est pas totale, mais partielle. Elle n'est totale et véritablement autoconstitutive que pour Dieu.

Ce faisant, Descartes toumait le dos à l'avertissement de Suarez, pour qui le per se, opposé à l'in alio, caractérise la substance en général, conformément à la doctrine du traité aristotélicien des Catégories, et fait donc abstraction de la question de la crétion. ${ }^{7}$ Per se et in alio caractérisaient le statut ontologique respectif de la substance et de l'accident. Avec Descartes, per se est interprété comme a se et in alio devient synonyme de ab alio. Al'absence ou à la présence d'une dẻpendance est substituce l'absence ou la présence d'une causalité extrinsèque et l'absence de causalité extrinsique cst comprise, en un sens positif, comme auto-causalité de la catsa sui, et ceci derechef contre l'avertissement de Suarez, qui, lorsqu'il lui arrivait de parler du caractère a se du principe (qu'il appelait aussi $e x$ se), s'abstenait de l'interpréter en un sens positif comme causa sui, mais l'entendait négativement comme le fait que, ctant parfait, le principe n'a pas besoin d'une cause. Au contraire, Descartes réduit le per se à l'a se ou, ce qui revient au même, interprite le per se comme a se ${ }^{8}$ et il entend clairement l'aséité comme auto-causalité.

6 On ne peut done souscrice à l'affismation de J.-L. Marion : "Descartes reditinit le concept de substance en sorte de luj ouvrir le seul champ du crée" (La theologie blanche de Descartes, p. 115).

7 Disput. metcph, XXXV, s. 1, n. 4. cf. Marion, Thiol. Blanche, p. Ilt.

8 Cf. le texte cité plus loin à la note 11. 
On pourrait donc penser que la définition de la substance donnée dans les Principes conduit à l'insupportable aporie qu'un concept qui était destiné à penser la réalité ontologique des réalités créées, âme et corps, s'avère ne valoir proprement que pour Dieu et valoir seulement de façon équivoque pour la créature. D'où le recours à une autre définition de la substance. qui est présentée expressément comme telle dans un texte légèrement antérieur aux Principes, mais postérieur aux premières mentions d'une définition "causasuiste" de la substance, que l'on trouve dès 1641 dans les Réponses aux IVes Objections d'Arnauld. Cette autre définition est la définition V de l'Exposé géométrique qui clôt les Réponses aux Secondes Objections: on appelle substance "toute chose dans laquelle réside immédiatement comme dans son sujet ou par laquelle existe quelque chose que nous concevons, c'est-à-dire quelque propriété, qualité ou attribut dont nous avons en nous une réelle idée". On remarquera que la définition de la substance n'arrive qu'en cinquième position dans la série des définitions, après les définitions de la pensée, de l'idée et des réalités objective et formelle de l'idée. Descartes commence par définir un attribut, la penséc, et la forme actuelle de la pensée qu'est l'idée, avant de se demander ce que peut être la substance dont cet attribut est l'attribut.

De fait, la substance est définie à partir de l'attribut comme le sujet de cet attribut ou ce par quoi existe cet attribut. Au premier abord, on retrouve ici la délinition aristotélicienne de l'ousia comme hypokeimenon, mais avec cette différence que l'hypokeimenon n'est plus dit exister par soi, mais seulement dans la mesure où existe au moins un attribut pour le qualifier. La substance a besoin d'au moins un attribut pour subsister. Car "nous n'avons point d'autre idće de la substance précisément prise, sinon qu'elle est une chose dans laquelle existe formellement ou éminemment ce que nous concevons". Certes l'altribut a besoin en un certain sens d'un sujet réel pour être lui-même réel: la lumière naturelle nous enseigne que le néant ne peut avoir aucun attribut réel"(ibid., déf. V). Mais on pourrait se demander pourquoi l'attribut ne pourrait se suftire à lui-même. De ce que le néant ne peut avoir d'attribut, il ne s'ensuit pas que l'attribut suppose nécessairement un sujet, mais seulement que le sujet de l'attribut, s'il y' a un sujet de l'attribut, ne pcut être qu'un sujet réel.

Il est vrai que l'attribut est la ratio cognoscendi de la substance. "Nous ne connaissons pas la substance immédiatement par elle-mème, mais seulement parce qu'elle est le sujet de quelque acte". Mais la question reste ouverte de savoir si la substance est la ratio essendi de l'attribut. Certes, il est dit par Descartes que la substance n'est pas seulement ce dans quoi, mais ce par quoi existe quelque chose que nous concevons. C'est la reprise textuelle du lexte d'Aristote, Mésaph., Z, 1, 1028 a 29, du moins tel qu'il est compris par Guillaume de Moerbeke: "Bonum aut sedens non sine hoc (subjectum) dicitur. Palam ergo quia propter eam (substantiam) et illorum singula sunt". Mais on ne voit pas comment le "ce sans quoi" (hoc sine quo non) dont parle effectivement Aristote serait en meme temps le "ce par quoi" (hoc propter quo) au sens d'une détermination causale (Aristote emploie la préposition dia, qui désigne un intermédiaire et n'a pas de sens causal). Dans un cas, il s'agit d'un support, dans l'autre, d'une cause. Mais les deux fonctions coïncident si la substance n'est autre que l'auto-position de l'attribut qui se précède dans l'être sous forme d'une condition minimale de possibilité. Ainsi la substance n'a-t-elle d'autre fonction chez Descartes que de permettre à l'attribut d'exister. Alors que pour Aristote une substance peut exister sans attributs puisqu'elle est déjà déterminée par elle-mêne (un homme sans qualités, etc., le meilleur exemple en étant Dieu lui-même, à qui l'on ne peut attribuer qualité, quantité, relations, etc.), la substance cartésienne ne peut exister sans un attribut, puisqu'elle n'est que la condition de possibilité de cet attribut. 
Cette thèse n'a, à vrai dire, de sens que dans la mesure où l'attribut, qui est conıu en premier, se confond quant à son contenu avec une substance qui ne lui préexiste que par une sorte de projection rétrospective et qui n'a pas d'autre raison d'être que de le rendre possible... De fait, l'attribut que Descartes envisage ici n'est pas n'importe quel attribut, mais l'attribut essentiel, i. e. ce qui se rapporte "immédiatement" au sujet, comme Descartes s'exprinte à plusieurs reprises. Ce que Descartes appelle "accident" n'est pas exclu pour autant, mais n'entrent dans la définition de la substance que ce type d'accidents qui sont impliqués par l'attribut essentiel et qui conviennent entre eux et avec lui, comme, par exemple, la grandeur, la figure et le mouvement par rapport à l'étendue), autrement dit ce qu'Aristote appelle "accidents par soi". L'attribut qui a besoin de la substance n'est done autre que l'essence méme de cette substance: par exemple, la pensée pour l’äme et l'étendue pour le corps. En ce sens, la substance est bien Ia "raison comniune" des attributs (Réponses aux Premières Objections), l'essence qui les fonde en tant qu'ils sont des attributs essentiels. Cette identification de la substance avec l'essence, dont elle n'est que la condition récurrente de possibilité sans autre contenu que l'essence elle-même, permet à Descartes d'établir ce qui l'intéresse véritablement: la distinction des substances entre elles et, en particulier, la distinction de l'àme et du corps.

Descartes pose deux criteres pour cette distinction: sont distinctes entre elles deux choses dont j'ai une idée claire et distincte; sont distinctes entre elles deux choses dont chacune est conçue séparément comme une chose complète, c'est-à-dire comme une substance. İn réalité, ces deux critères n'en font qu'un. Je ne peux avoir d'idée claire et distincte d'une substance qui se dissimulerait comme substrat sous-jacent, objet $=X$, derrière ses attributs. Je ne peux avoir d'idée claire et distincte que d'un attribut. Mais l'idée claire et distincte de l'attribut permet de concevoir comme chose complete, i. e. comme substance, le sujet de l'attribut si celui-ci n'est pas autre chose que l'hypostase de cet attribut. Ainsi est prouvée la distinction réclle de l'âme et du corps: l'âme et le corps sont des choses réellement distinctes, puisque nous concevons comme réellement distincts les attributs essentiels dont ils sont les substances. Sans la mise en correspondance de l'attribut essentiel et de sa substance, rien ne s'opposerait à ce que deux attributs, comme la pensée et l'étendue appartiennent au mème sujet.

La critique qui est généralement adressée d Descartes est que les conséquences de son raisonnement en outrepassent les prémisses. On lui accorde, s'agissant de la pensée, qu'il n'y a pas d'acte de pensée sans un "je" qui pense. Mais cette exigence logique, en quelque sorte grammaticale - pas d'attribut sans sujet - n'autorise pas l'hypostase du sujet logique en un sujet réel, substantiel. Descartes commet ce que Kant appellera le paralogisme de la substantialité. En effet, pourquoi poser une substance derrière les attributs, si la substance n'est pas autre chose que l'essence, c'est-ì-dire l'unité des attributs essentiels ou, comme dit Descartes l'acte qui révèle l'essence? Les attributs ont besoin d'un sujet logique, mais non d'un sujet réel qui sernit distinct d"eux.

Elie Rabier, un auteur de la fin du xixe siècle aujourd'hui bien oublié, formule bien ce type de critique. ' L'argumentation de Descartes en faveur de la distinction de l'âme et du corps présuppose que la distinction des attributs dont nous avons des idées distinctes - pensée et ćtendue- implique la distinction des substances correspondantes: La conclusion n est légitime qu'à la condition d'identifier substance et attribut à chaque fois essentiel (sinon, il pourrait s'agir des attributs d'une mêne substance). Il taut donc admettre que "toute la nature de l'es-

9 E. Rabier, éd. du Discours de la Mfithode, Paris, Delagrav'e, 1894. 
prit consiste sculement à penser" et que "toute la nature du corps consiste seulement en ce point que le corps est une chose étendue" (Réponses aux lVes Objections). Mais alors la substance n'est-elle pas un redoublement inutile de l'essence? "Ainsi c'est une chimère, écrit Rabier, d'imaginer un substratum de la pensée fermé lui-même à la pensée, un je ne sais quoi qui pense et qui ne se pense point". Si Descartes n'est pas parvenu à formuler correctement cette conséquence, dont la reconnaissance critique et le rejet en auraient fait le précurseur de l'idéalisme transcendantal, c'est, estime Rabier, à cause de "l'importunité de souvenirs d'école", à la "gêne d'un langage fait avec la théorie contraire".10

Il y a une certaine vérité herméneutique dans ce jugement. Les souvenirs d'école sont plus agissants chez Descartes qu'on ne l'a longtemps pensé, mais qu'il doive contraindre sa pensée à adopter le langage d'une théoric contraire, on n'ira pas jusqu'à l'aflirmer, ne serait-ce que parce que Descartes ne pouvait percevoir l'incompatibilité avec la sienne d'une théorie ontologique dont il avait emprunté les concepts et mème les définitions, mais en subvertissant leur sens. L'emprunt et la transformation, la continuité et le changement, caractérisent l'usage que fait Descartes du concept aristotélicien de substance.

Les deux définitions cartésiennes de la substance sont l'une et l'autre empruntées à Aristote à travers la scolastique, en particulier suarézienne. L'emprunt est littéral pour la première, plus indirect pour la seconde, mais pourrait être assez aisément reconstitué. La première se rélêre à l'auto-suffisance du sujet sans les atıributs:, la seconde à la nécessité pour les attributs, y compris ceux qui disent l'essence, d'avoir ce mêtne sujet pour substrat. Substantia sub-sistir et sustinet. Les deux définitions cartésiennes, comme les deux caractérisations aristotéliciennes, ne sont pas contradictoires entre elles, mais complémentaires. Elles correspondent à une double exigence d'auto-suffïsance (choriston) et de déterminité (tode ti). Mais chez Aristote ces deux exigences restent disjointes et concurrentes: le sujet aristotélicien n'est pas entièrement déterminé par ses altributs ni même par l'attribut essentiel qu'est sa quiddité; il est lesté par la matière, qui repressente une réserve inépuisable d'accidentalité et d'indétermination. La question ti esti ou même ti en einai ne permet de répondre que de façon générale à l'exigence de détermination du sujet. La forme essentielle est bien la cause de l'einai (ousia ... aition proton tou einai, Métaph., Z, 17, 1041 b 28), mais il faut l'entendre au sens où elle détermine l'àtretel du sujet, le Sosein, non le Sein, l'existence qui demeure indémontrable et indéductible. Descartes, selon une pente qui est naturelle à l'intelligence, mème si elle $n$ ' est pas entièrement fidèle à la chose elle-méme, live ces obstacles, ces limitations. De ce point de vue, les deux détinitions cartésiennes convergent.

Dans la première définition, l'auto-suffisance devient auto-détermination, auto-position de l'essence. Dans un texte remarquable, justement souligné par J.-L. Marion, Descartes donne lui-mème cette explication de la causa sui: "esse sui causam, hoc est, esse per se, nec aliam habere causam quam propriam suan essentiam, qual dici posset causa formalis"." La substance, entendue comme cause formelle, est la raison non seulement de ses attributs, mais de sa propre existence. Dans la deuxième définition, qui part de l'attribut pour remonter au sujet, nous voyons que celui-ci n'est pas autre chose que l'auto-position de l'attribut en tant qu'il est attribut essentiel. La substance devient ainsi la raison de sa propre essence. Dans les deux cas, on assiste à un processus d'auto-fondation qui manifeste le pouvoir désonnais sans partage de la représentation claire et distincte. Le véritable subjectum n'est plus, en dépit d'une apparen-

10 Rabier, op. cit., p. 109.

II Lettre d X, 16+0 ou 16+3 ?, AT, t. V. p. 5\$6, 6-11. 
ce qui peu à peu se dissout, ce qu'il y a de sous-jacent, le substrat des attributs, mais le sujet au sens moderne, l'ego de l'ego cogito, qui nést dit res que par une dernière concession à Suarez et "substance" que par une demière concession à Aristote, mais en un sens qui, à la littéralité près, ne doit presque plus rien à la substance aristotélicienne. On assiste chez Descartes d l'auto-dissolution de la substance comme sujet, consécutive a son essentialisation. On assiste parallèlement au dẻplacement du sujet, qui n'est plus à chercher dans la chose extéricure, mais dans le sujet de la représentation.

Avec Descartes, la métaphysique moderne de la subjectivité succède à l’ontologie de la substantialité, l'univocité du représentable, réléré à l'absoluité du sujet pensant, supplante la plurivocité de l'àtre aristotélicien.ll est aujourd'hui trivial de reconnaitre là une nutation décisive dans l'histoire de la pensée et de fêtre, peut-être même dans f'histoire tout court. Mais pour situer le lieu exact de celte mutation, il n'est sans doute pas inutile de mesurer avec précision la transformation, au premier abord presque inapparente, que connaissent chez Descartes les concepts ontologiques hérités de la tradition. 